\title{
Emergent Repair of Cardiac Rupture and Successful AF Ablation Procedure
}

\author{
Sahin Iscan*, Bortecin Eygi and Koksal Donmez \\ Department of Cardiovascular Surgery, Izmir Ataturk Education and Research Hospital, Katip Celebi \\ University, Izmir, Turkey
}

*Corresponding author: Sahin Iscan, Department of Cardiovascular Surgery, Katip Celebi University, Izmir Ataturk Education and Research Hospital, Izmir, 35360, Turkey, Tel: 0090-5054-8820-90, E-mail: sahiniscan@hotmail.com

\begin{abstract}
Today, ablation techniques for atrial fibrillation (AF) are successfully increasing but catheter ablation may have important complication risks. We report the case of a 56-year-old woman with chronic AF who had left atrial rupture during catheter ablation. Echocardiography showed pericardial effusion during procedure and she was operated immediately. Left atrial rupture repaired with sternotomy. After stabilizing the hemodynamic and metabolic parameters, intraoperative bipolar ablation was performed. Patient had sinus rhythm postoperatively and she still has after 1-month follow-up. Percutaneous catheter ablation may have fatal complication risk and surgeon should always be courageous and prepared for ablation surgery, even in unusual cases.
\end{abstract}

\section{Keywords}

Atrial fibrillation, Ablation, Cardiac rupture

\section{Introduction}

Today, ablation strategies, like percutaneus and surgical ablating techniques, are successfully increasing for treating of AF. These methods are accepted safe for symptomatic AF patients. Success rates of catheter ablation vary between $50 \%$ and $80 \%$, for paroxysmal and persistent nonvalvular AF [1]. In cardiovascular practices, using of surgical ablation techniques increased in addition to valvular repair or valve replacement surgery. Success rate of surgical ablation vary between $40 \%$ and $70 \%$ with coronary and valvular cardiac diseases [2]. In the light of these results, patients benefit from ablation techniques and patients are protected from AF induced cardiac remodeling in long term period with successful AF ablation.

Catheter ablation for AF may have complication risks, like stroke $(<1 \%)$, atriooesophageal fistula and late pericardial tamponade [3]. In this case, we wanted to present an acute pericardial effusion due to catheter ablation procedure, its emergent repair and completing of percutaneous AF ablation procedure with surgical bipolar ablation.

\section{Case Report}

56-years-old female patient was admitted to hospital with chronic AF. Percutaneous radiofrequency ablation procedure was planned for treatment of AF. Patient was taken to catheterization laboratory. $1 \mathrm{~cm}$ long pericardial effusion was detected during the ablation procedure. A pericardial drainage catheter was inserted with subxifoid technique guiding with echocardiography. Pericardial drainage and autotransfusion were provided by cardiologist. Anticoagulation level of the patient was reversed with protomin. Patient was followed in the angiography unit with monitorization and echocardiographic imaging. After the arrival of cardiovascular surgery consultant, pericardial effusion of the patient increased to $2 \mathrm{~cm}$ long, but there was no cardiac tamponade. We decided to operation after 30 minutes from the first cardiac rupture. Patient was taken to operating room to prevent cardiac tamponade. Cell saver device was prepared, and median sternotomy was performed. Pericard was opened and there was $1 \mathrm{~cm}$ long left atrial rupture with serious bleeding. Rupture area was controlled, and it was sutured with prolen pledget sutures. After stabilization of patient hemodynamic and metabolic parameters (blood gases, hematocrits and etc.), we decided to perform bipolar ablation procedure due to AF. Right superior and right inferior pulmonary veins

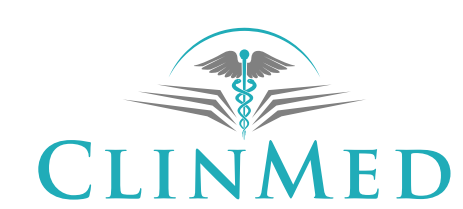

INTERNATIONAL LIBRARY
Citation: Iscan S, Eygi B, Donmez K (2018) Emergent Repair of Cardiac Rupture and Successful AF Ablation Procedure. Int J Clin Cardiol 5:111. doi.org/10.23937/2378-2951/1410111 Received: March 28, 2018; Accepted: May 08, 2018; Published: May 10, 2018

Copyright: (c) 2018 Iscan S, et al. This is an open-access article distributed under the terms of the Creative Commons Attribution License, which permits unrestricted use, distribution, and reproduction in any medium, provided the original author and source are credited. 
were explored from the posterior side of interatrial septum and they were separated from surrounding tissues. Left superior and inferior pulmonary veins were also explored and they were separated from surrounding tissues by elevating the left ventricle apex slightly. In this time, activated clotting time was 180 seconds. Right superior and inferior pulmonary veins were carefully ablated using bipolar ablating device. Later, left ventricle was slightly elevated again and left superior pulmonary vein, left inferior pulmonary vein and left atrium auricle were ablated. Due to the fact that it was a beating heart procedure care was taken to the hemodynamic indicators of patient during the ablation procedure. From the anterior side of interatrial septum, a purse string suture which is in the middle of the plane between vena cava superior and inferior was placed to right atrium. Right atrium was opened. Bipolar ablation device was placed from this incision and right atrial ablation was performed toward vena cava superior, vena cava inferior and right atrium auricle. Ablation device was taken out from the right atrium and purse suture was ligated. Finally, right atrial auricle was ablated, and ablation procedure was completed. After that, amiodarone perfusion was started, and it was continued 2 days. Patient had normal sinus rhythm after completing the ablation procedure. Patient was externed with sinus rhythm on postoperative fifth days. In the 3 mounts follow up, patient still had sinus rhythm.

\section{Discussion}

Cardiac perforation related to catheter ablation is an infrequent complication, but it may be fatal. Its incidence is $0.2-6 \%$. Left atrium, right atrium and apex of right ventricle are the most seen areas of perforation [4]. In this case, we detected a $1 \mathrm{~cm}$ long rupture area in left atrial appendage. Mechanical catheter manipulation and excessive tissue ablation are the important risk factors of rupture during ablation procedure [5]. Pericardiocentesis with fluoroscopy or echocardiography guided subxiphoid approach is the first therapeutic approach for a cardiologist. Its effectiveness is limited, and it may be fatal if there is a long rupture area. Small rupture areas may be controlled with this technique, but patients must be followed in intensive care unit with monitorization and frequent echocardiographic control to avoid pericardial tamponade. Studies showed that $13 \%$ of patients after atrial perforation and $55 \%$ of patients after ventricle perforation needed to surgical intervention for catheter ablation caused cardiac ruptures [6]. In our patient, we detected $2 \mathrm{~cm}$ pericardial effusion without cardiac tamponade, 30 minutes later of the first rupture time. We take the patient to the operating room as fast as to prevent cardiac tamponade and cardiac arrest. In this period, autotransfusion may be helpful to delay tamponade for cardiologists with increased risk of inflammation due to pericardial contact of the blood. But the use of cell-salvage system is the best as we used during operation. In our patient we saw a $1 \mathrm{~cm}$ long rupture in left atrial appendage with serious bleeding and we repaired the rupture.

Discontinuation of AF ablation procedure due to cardiac tamponade is a disappointment for physicians. For patients who don't need surgical intervention, it means a repeat ablation procedure and it is also a burden for patient and physician. For patients who need to surgical intervention due to cardiac tamponade, it is an unexpected death risk. This complication also has a tragic aspect for a patient who looks for a better cardiac health. In this case we successfully repaired rupture area and after the stabilization of the patients' hemodynamic and metabolic parameters, we decided to perform bipolar ablation procedure to the patient for preventing these risks both patient and physician. When we searched to the literature, this is the first surgical ablation report to gather with the repair of a fatal left atrial rupture after a complicated percutaneous catheter ablation procedure.

In conclusion, surgical ablation technique is widely in use and we frequently perform AF ablation with other surgical interventions of cardiac diseases as cardiovascular surgeons. This case enabled us to unconventionally complete an incomplete percutaneous ablation procedure. This case made us to think that we may prefer surgical ablation techniques more frequently and boldly appropriately selected patients.

\section{Compliance with Ethical Standards}

This study was not funded by any company. Author declares that he has no conflict of interest.

\section{Ethical Approval}

This article does not contain any studies with human participants performed by any of the authors.

\section{Informed Consent}

Informed consent was obtained from individual participant included in the study.

\section{References}

1. Sultan A, Lüker J, Andresen D, Kuck KH, Hoffmann E, et al. (2017) Predictors of Atrial Fibrillation Recurrence after Catheter Ablation: Data from the German Ablation Registry. Sci Rep 7: 16678.

2. Beşir Y, Gökalp O, Yetkin U, Çelik E, Iner H, et al. (2015) Comparison of amiodarone and propafenone for maintenance of stable sinus rhythm after bipolar radiofrequency ablation combined with a mitral valve procedure in patients with mitral valve disease and persistent atrial fibrillation. Türk Göğüs Kalp Dama 23: 258-263.

3. Kirchhof P, Benussi S, Kotecha D, Ahlsson A, Atar D, et al. (2017) 2016 ESC Guidelines for the Management of Atrial Fibrillation Developed in Collaboration With EACTS. Rev Esp Cardiol (Engl Ed).70: 50.

4. Cappato R, Calkins H, Chen SA, Davies W, lesaka Y, et al. (2009) Prevalence and causes of fatal outcome in catheter ablation of atrial fibrillation. J Am Coll Cardiol 53: 1798-1803. 
5. Mujović N, Marinković M, Marković N, Kocijančić A, Kovačević V, et al. (2016) Management and Outcome of Periprocedural Cardiac Perforation and Tamponade with Radiofrequency Catheter Ablation of Cardiac Arrhythmias: A Single Medium-Volume Center Experience. Adv Ther 33: 1782-1796.
6. Bunch TJ, Asirvatham SJ, Friedman PA, Monahan $\mathrm{KH}$, Munger TM, et al. (2005) Outcomes after cardiac perforation during radiofrequency ablation of the atrium. J Cardiovasc Electrophysiol 16: 1172-1179. 